\title{
First in vivo pulmonary Carcinosarcoma in a cat
}

\author{
Marisa Santos*, Marianne Heimann, Johan Caraty, Alexandre Bongartz
}

粦BSAVA

\section{INTRODUCTION}

Carcinosarcoma is a malignant tumour containing both carcinomatous and sarcomatous elements ${ }^{1}$. Primary pulmonary carcinosarcoma is rare in humans accounting for less than $1 \%$ of thoracic cancers ${ }^{2}$ and is extremely rare in animals ${ }^{3-4}$

Diagnosis is based on morphology and immunohistochemistry, with presence of 2 malignant cell populations ${ }^{5}$

Surgery provides the most substantial chance at remission and submission for histopathology leads to a complete diagnosis of carcinosarcoma. Surgery is the preferred first line of treatment for human patients with non-metastatic disease

There are still many questions to be answered about the pathogenesis, behaviour and the prognosis of this uncommon neoplasm, rarely reported in Veterinary Medicine.

\section{OBJECTIVE}

To report the first case of an in vivo pulmonary carcinosarcoma diagnosed in a cat and provide data that may be of value for treatment decisions of pulmonary carcinosarcoma and subsequent prognosis in cats.

\section{CASE DESCRIPTION}

A male neutered domestic shorthair cat, 20-year-old, BSC 4/9 was referred for investigation of a non-productive cough and chronic weight loss

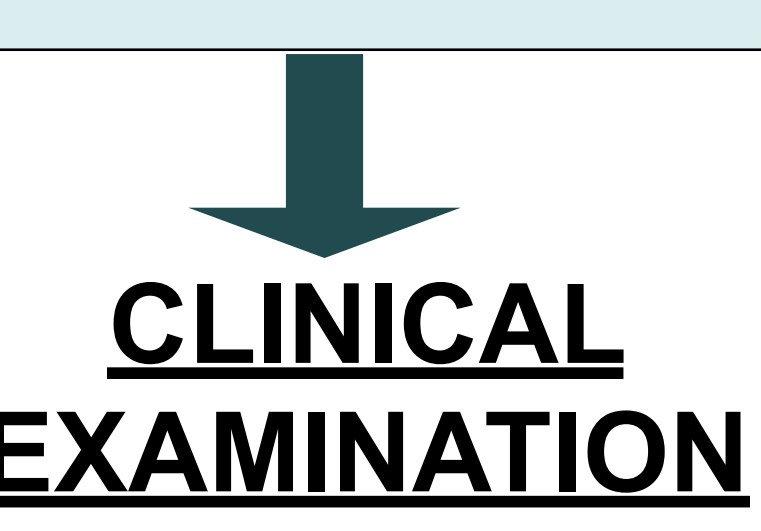

Unspecific

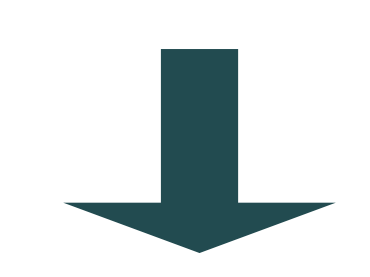

Thoracic radiography

Rounded, soft tissue opacity of right-sided pulmonary caudal lobe

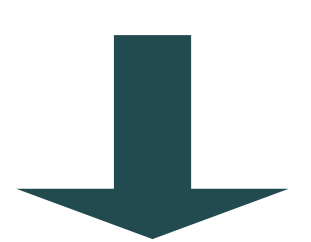

CT-scan**

Section thickness $0.65-1.5 \mathrm{~mm}$

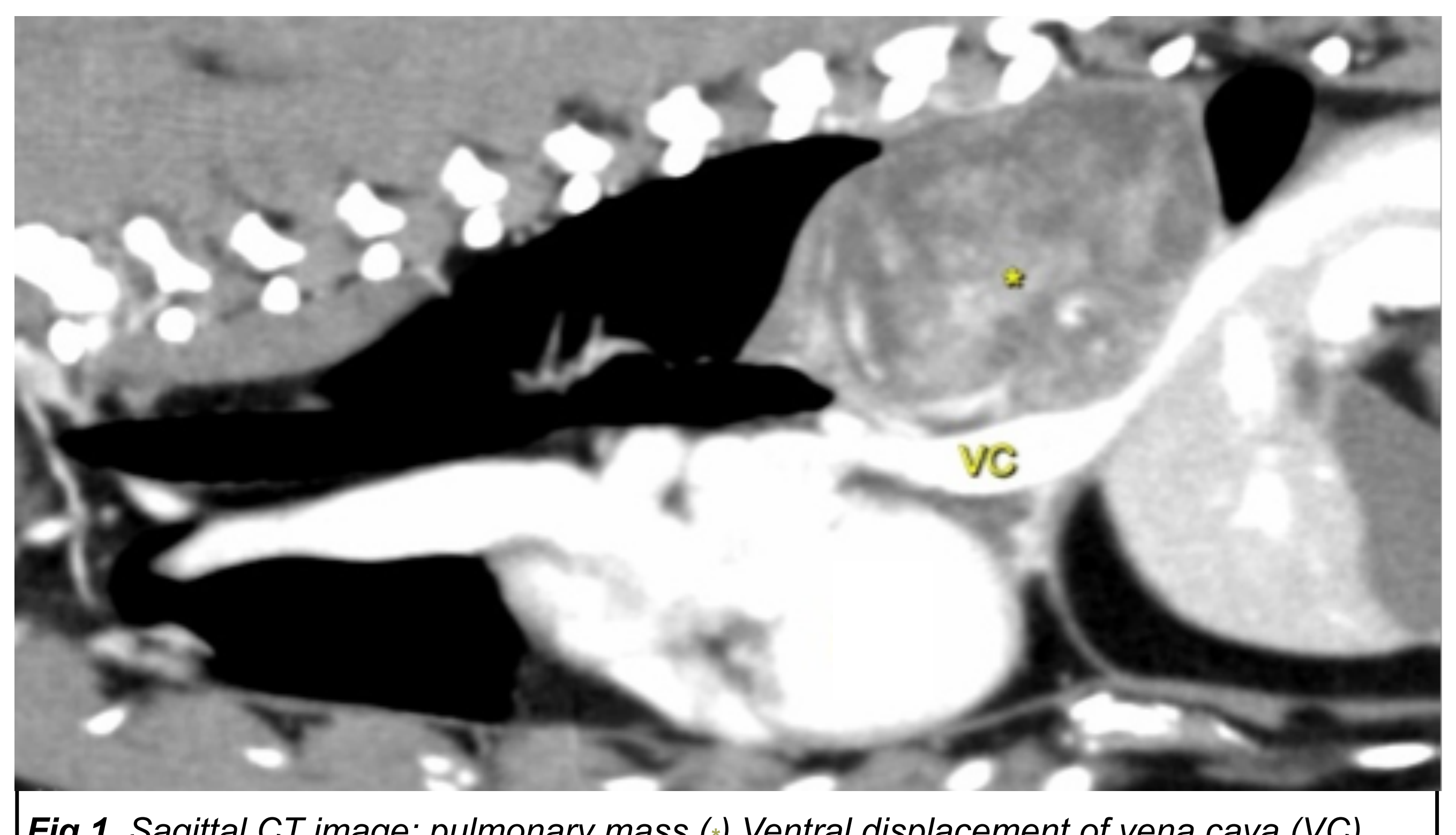

\section{1}

Cavitary, $\overline{4.5 \times \overline{5} .5 \times 3} \overline{\mathrm{cm}} \mid \quad \overline{\mathrm{N}} \mathrm{o}$ tracheobronchial soft-tissue mass onl lyymph node enlargement the right caudal lungl INo intrapulmonary lobe I Imetastasis

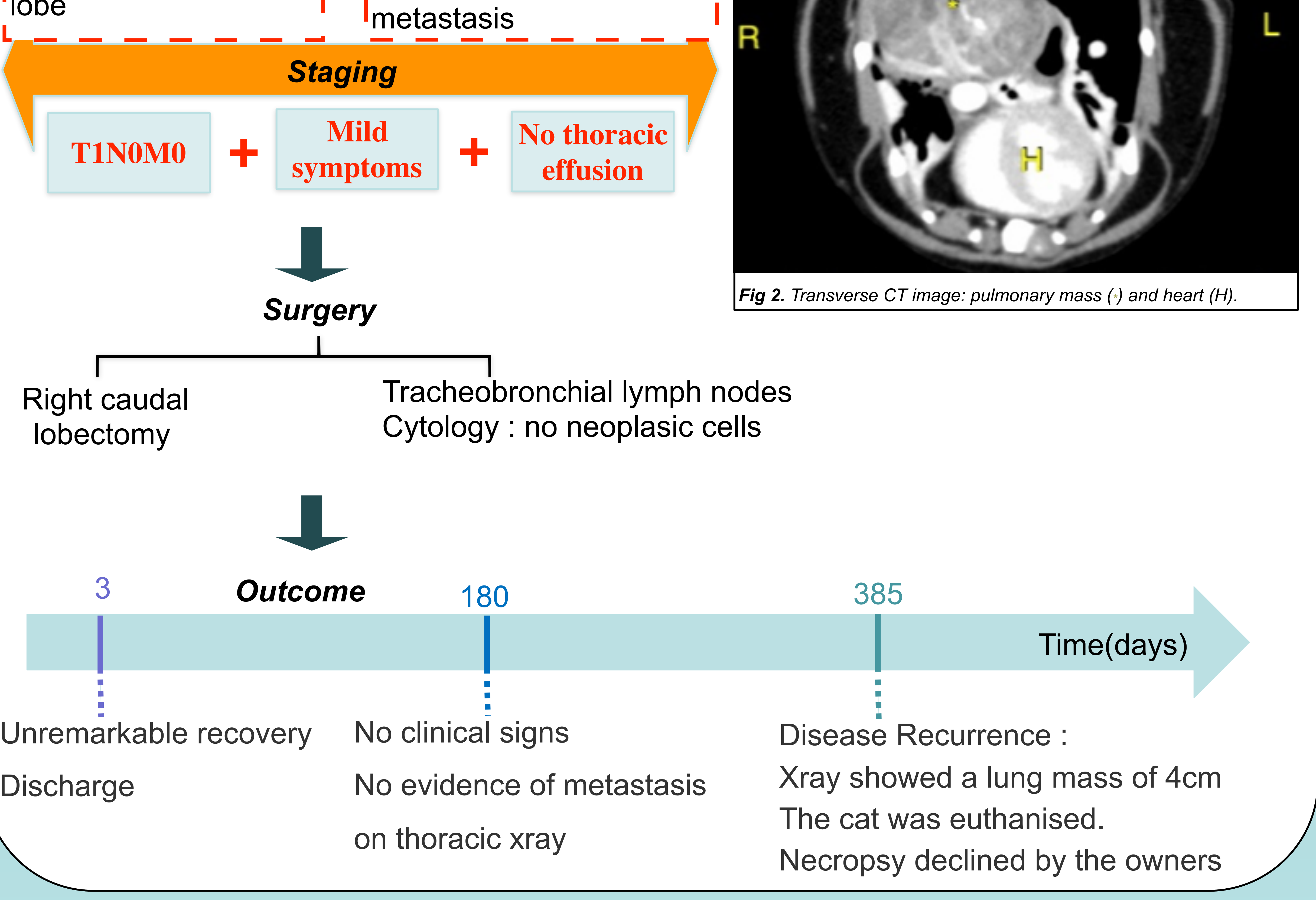

\begin{tabular}{llll|}
\hline \multicolumn{4}{c|}{ REFERENCES } \\
1. Ersek et al. 2019) & 3. Salas et al. 2002 & 5. Sapierzynski et al. 2018 & ${ }^{*}$ marisa. santos.mail@gmail.com \\
2. Travis et al. 2015 & 4. Ghisleni et al. 2003) & 6. Maritato et al. 2014 & ${ }^{*} \mathrm{CT}$ : Toshiba Aquillon 64 slices
\end{tabular}

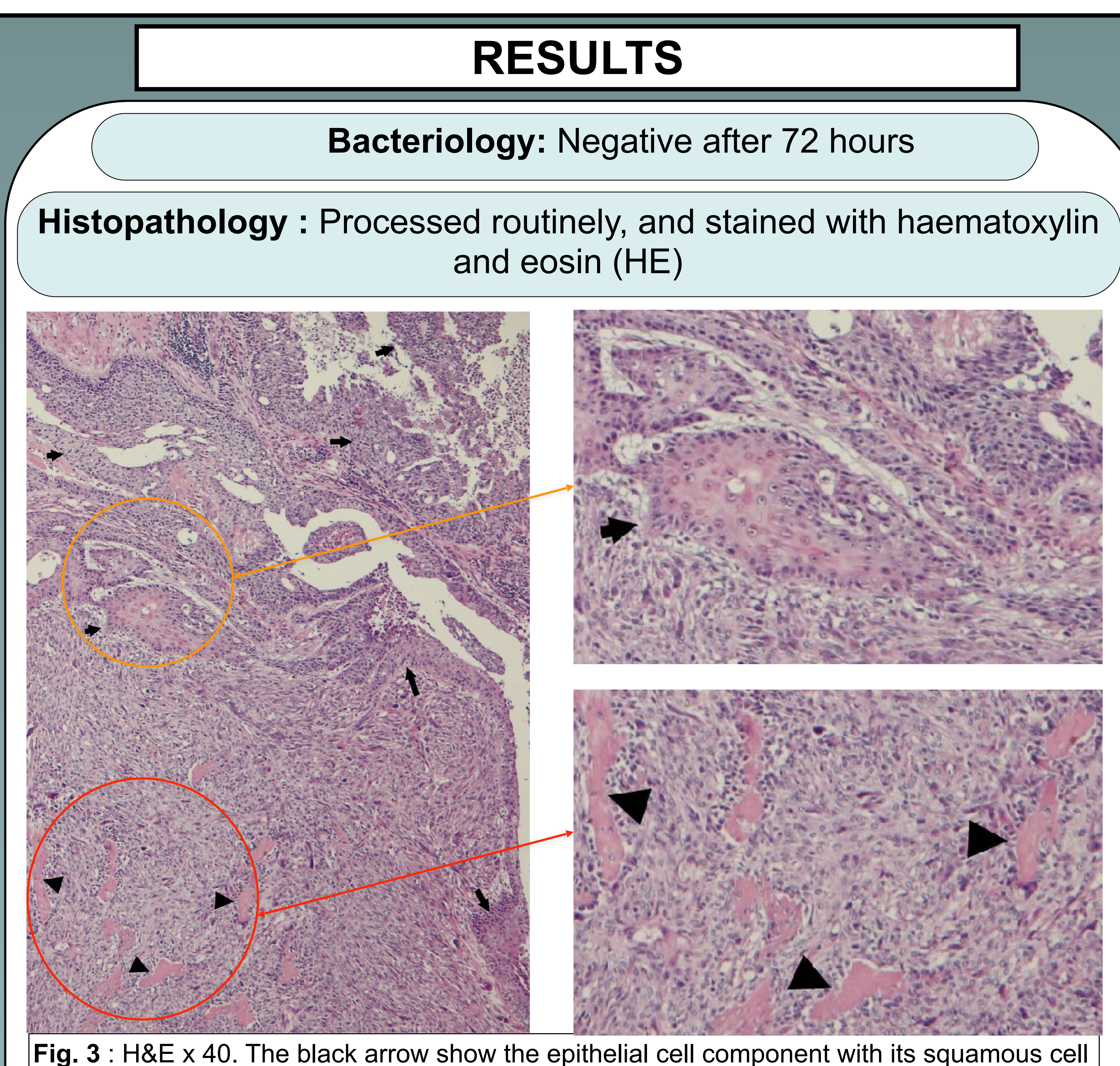

Fig. 3 : H\&E x 40. The black arrow show the epithelial cell component with its squamous cell shape. The black arrow head identify the mesenchymal component with bone trabeculae.

Carcinosarcoma suspected based on the identification of $\overline{2}$ different cell populations

Immunohistochimy: Vimentine and Cytokeratin

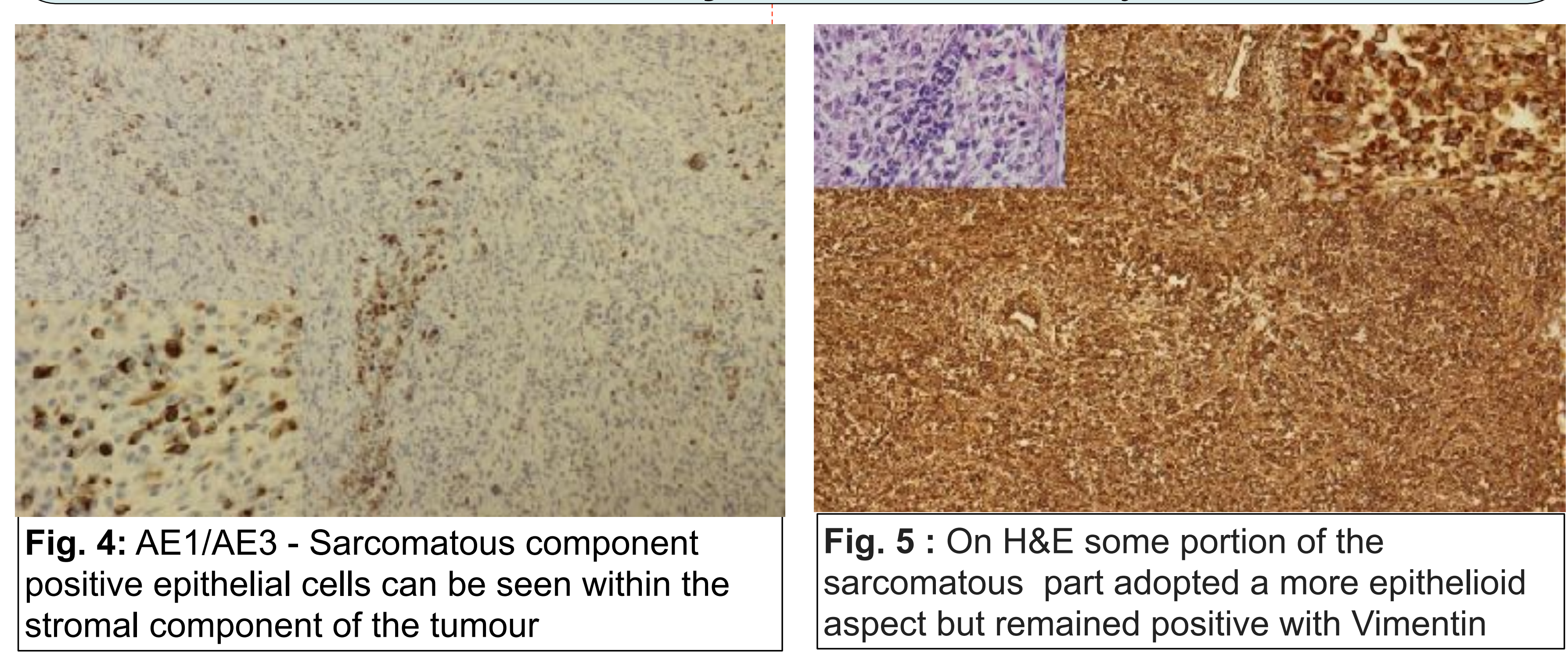

\section{Definitive Diagnosis}

Immunohistochemistry confirmed the presence of 2 distinct tumour components: an epithelial population, mainly of papillary architecture and a foci of squamous metaplasia, positive to cytokeratin and a sarcomatous population that appeared mainly as spindle cells with crisscrossing fascia formation, positive to vimentin

\section{DISCUSSION}

Carcinosarcomas have been rarely reported in Veterinary, with only 2 cases of pulmonary carcinosarcoma diagnosed at post mortem examination, in veterinary literature ${ }^{3-4}$

This is the first report in vivo of a successful treatment of pulmonary carcinosarcoma with a overall survival time (OS) of 12 months.

The sporadic cases of feline carcinosarcoma diagnosed in another anatomic locations, revealed a poor outcome, with high rate of metastases documented at the moment of diagnosis or shortly after.

Based on human literature, (OS for surgery alone: 20 months > combined surgery and radiation: OS 7 months $>$ radiation alone: OS 4 months) $)^{4}$ surgical resection was the only treatment that this cat underwent

The 12 month survival time is within the previous data reported by Maritato et al. in cats with pulmonary neoplasms staged T1NOMO

We believe that a localised tumour, with mild symptoms and no malignant thoracic effusion could explain the acceptable outcome and are the most contributive factors to OS, instead of the aggressive nature of this cancer.

\section{STATEMENT}

Although feline Carcinosarcoma is rare, it should be considered a differential diagnosis of primary lung neoplasias. Surgical treatment could lead to acceptable outcome if negative staging is observed in CT examination. 